\title{
CONDITIONS FOR SOME POLYGONAL FUNCTIONS TO BE BAZILEVIČ
}

\author{
B. A. CASE AND J. R. QUINE
}

\begin{abstract}
Univalent functions in the disc whose image is a particular eight-sided polygonal region determined by two parameters are studied. Whether such a function is Bazilevič is determined in terms of the two parameters, and the set of real $\alpha$ 's is specified such that the function is $(\alpha, \beta)$ Bazilevič for some $\beta$. For any interval $[a, b]$ where $1<a \leqslant 3 \leqslant b$, a function of this type which is $(\alpha, 0)$ Bazilevic precisely when $\alpha$ is in this interval is found. Examples are given of non-Bazilevic functions with polygonal images and Bazilevič functions which are $(\alpha, 0)$ Bazilevič for a single value $\alpha$.
\end{abstract}

Introduction and notation. For $\alpha>0$ and $\beta$ real, let $B(\alpha, \beta)$ be the class of $(\alpha, \beta)$ Bazilevič functions introduced in [2]. Sheil-Small [7] established that $\{(\alpha, \beta) \mid \alpha>0$, $f \in B(\alpha, \beta)\}$ is a closed, convex subset of the half-plane $\alpha>0$. Campbell and Pearce [3] refer to this set as the representation projection of the function, and we will refer to its intersection with the $\alpha$-axis as the $\alpha$-projection which, if nonempty, is a point or an interval. In this paper we look at the $\alpha$-projections for a two parameter family of functions $f\left(z ; \psi_{1}, \psi_{2}\right)$ mapping $|z|<1$ conformally onto the interior of a polygon whose geometry is determined by the two parameters. The $\alpha$-projection is given in terms of the endpoints of the interval as functions of the parameters $\psi_{1}, \psi_{2}$. Those $f\left(z ; \psi_{1}, \psi_{2}\right)$ for which $\psi_{1}>2 \psi_{2}$ are not in any $B(\alpha, \beta)$; i.e., we also have a subfamily consisting of non-Bazilevič functions. The type of non-Bazilevič function of Plaster [6] is a limiting function of the above subfamily. Our family also includes bounded functions whose $\alpha$-projection is a single point.

We now describe the functions $f\left(z ; \psi_{1}, \psi_{2}\right)$. Let $f\left(z ; \psi_{1}, \psi_{2}\right)$ be the unique univalent analytic function in $|z|<1$ (normalized $f(0)=0 ; f^{\prime}(0)>0$ ) with continuation to $|z|=1$ having a closed polygonal image $A_{0} A_{1} A_{2} \cdots A_{8}, A_{0}=A_{8}$ (Figure 1) determined by the angle parameters

$$
0<\psi_{1}<\pi / 2, \quad 0<\psi_{2}<\pi / 4
$$

as follows:

$$
\begin{gathered}
A_{5}=-1-i ; \quad A_{6}=1-i ; \quad A_{7}=1+i ; \quad A_{8}=A_{0}=-1+i ; \\
A_{1}=-1+i \tan \psi_{2} ; \quad A_{4}=\overline{A_{1}} ; \quad \arg A_{2}=3 \pi / 4 ; \\
\arg \left(A_{2}-A_{1}\right)=\psi_{1} ; \quad A_{3}=\overline{A_{2}} .
\end{gathered}
$$

Received by the editors December 22, 1981.

1980 Mathematics Subject Classification. Primary 30C45.

Key words and phrases. Univalent function, Bazilevič function.

(C)1983 American Mathematical Society $0002-9939 / 82 / 0000-1257 / \$ 02.75$ 
(The figure suggests that we refer to $f\left(z ; \psi_{1}, \psi_{2}\right)$ satisfying (1) and (2) as a keyhole function.) The exterior angles are denoted $e_{k}=\pi-\angle A_{k}$ in the usual manner (see Nehari [5, p. 188]). Hence, when $\angle A_{k}<\pi$, then $0<e_{k}<\pi$, and when $\angle A_{k}>\pi$, $-\pi<e_{k}<0$. The exterior angles have values

$$
\begin{gathered}
e_{1}=e_{4}=\pi / 2+\psi_{1} ; \quad e_{2}=e_{3}=-\pi / 2-\psi_{1} ; \\
e_{5}=e_{6}=e_{7}=e_{8}=e_{0}=\pi / 2 .
\end{gathered}
$$

A remark is appropriate about two of the cases for the limiting values of the parameters $\psi_{1}, \psi_{2}$. When $0<\psi_{2}<\pi / 4$ and $\psi_{1} \rightarrow 0$, the limit function is close-toconvex. For $0<\psi_{1}<\pi / 2$ and $\psi_{2} \rightarrow 0$, the limit function may be thought of as the type of non-Bazilevič function described by Plaster [6].

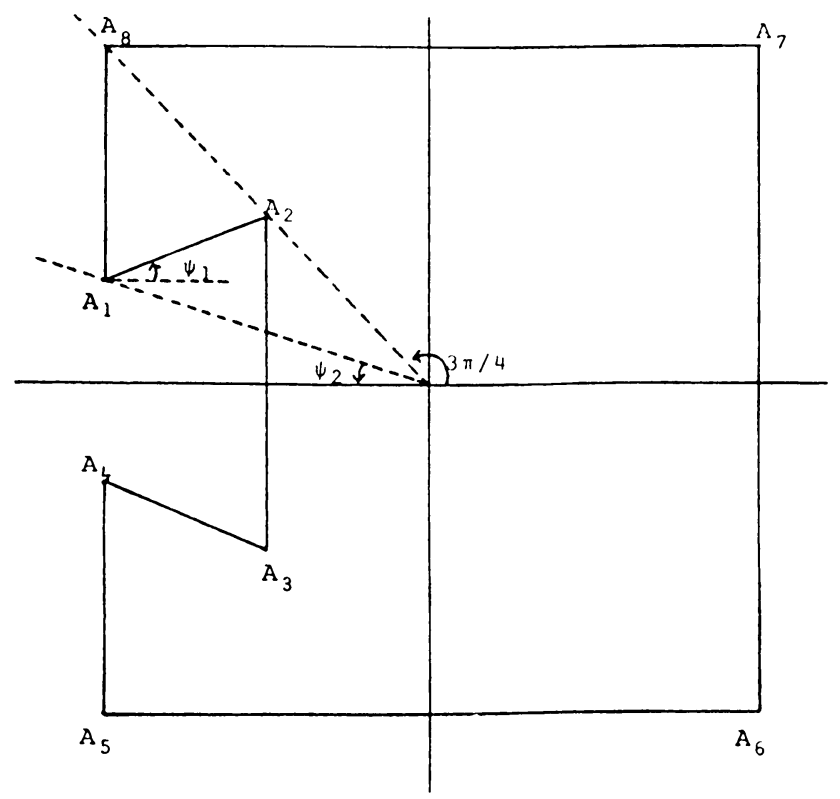

FIGURE 1

Some preliminary results. We will need the following theorem of Sheil-Small.

Theorem 1 (Sheil-SMALl). If $f(z) \in B(\alpha, \beta)$ then

(4)

$$
\int_{\theta_{1}}^{\theta_{2}}\left\{1+\operatorname{Re} \frac{r e^{i \theta} f^{\prime \prime}\left(r e^{i \theta}\right)}{f^{\prime}\left(r e^{i \theta}\right)}+(\alpha-1) \operatorname{Re} \frac{r e^{i \theta} f^{\prime}\left(r e^{i \theta}\right)}{f\left(r e^{i \theta}\right)}-\beta \operatorname{Im} \frac{r e^{i \theta} f^{\prime}\left(r e^{i \theta}\right)}{f\left(r e^{i \theta}\right)}\right\} d \theta>-\pi,
$$

for $0<r<1$ and $0<\theta_{2}-\theta_{1}<2 \pi$.

Conversely, if $f(z)$ is analytic in $|z|<1$, with $f(0)=0, f(z) \neq 0(0<|z|<1)$ and $f^{\prime}(z) \neq 0(|z|<1)$, and if $f(z)$ satisfies (4) for $0<r<1$ and $0<\theta_{2}-\theta_{1}<2 \pi$ when $\alpha \geqslant 0$ and $\beta$ is real, then $f(z)$ is univalent in $|z|<1$ and is in $B(\alpha, \beta)$ for $\alpha>0$. 
For convenience we denote the integrand of (4) as $F(f, \alpha, \beta ; z)$, and the entire left side integral as $I_{r}\left(\theta_{1}, \theta_{2}\right)$. We define, additionally, a function $J\left(\theta_{1}, \theta_{2}\right)$ for $0 \leqslant \theta_{2}-$ $\theta_{1} \leqslant 2 \pi$ :

$$
J\left(\theta_{1}, \theta_{2}\right)=\liminf _{\substack{r \rightarrow 1^{-} \\ \mu_{1} \rightarrow \theta_{1} \\ \mu_{2} \rightarrow \theta_{2}}} I_{r}\left(\mu_{1}, \mu_{2}\right) .
$$

Next we note a lemma which is needed to show that the representation projection of a keyhole function is symmetric in the $\alpha$-axis.

Lemma 1. If $f \in B(\alpha, \beta)$ then $\bar{f} \in B(\alpha,-\beta)$.

Proof. Using the elementary properties of conjugates, we see that $F(\bar{f}, \alpha,-\beta ; z)$ $=F(f, \alpha, \beta ; \bar{z})$ where $\bar{f}(z)=\overline{f(\bar{z})}$. The lemma now follows from Theorem 1 .

The next lemma shows that if the Sheil-Small condition with weak inequality holds in the limit as $r \rightarrow 1^{-}$, the functions $f\left(z ; \psi_{1}, \psi_{2}\right)$ are Bazilevič.

LeMma 2. If $f(z)$ is analytic in $|z|<1$, with $f(0)=0, f(z) \neq 0(0<|z|<1)$, $f^{\prime}(z) \neq 0(|z|<1)$, and $J\left(\theta_{1}, \theta_{2}\right) \geqslant-\pi$ for $0 \leqslant \theta_{2}-\theta_{1} \leqslant 2 \pi$, then $f(z)$ is Bazilevič.

Proof. For fixed $\phi \in \mathbf{R}$ define

$$
G_{\phi}\left(r e^{i \tau}\right)=I_{r}(\phi-\tau, \phi+\tau)=\int_{-\tau}^{\tau} F\left(f, \alpha, \beta ; r e^{i(t+\phi)}\right) d t
$$

where $0<r<1,0<\tau<\pi$. The function $G_{\phi}$ is harmonic. One way to see this is to compute the Laplacian in polar coordinates, differentiate under the integral sign, and then use the fact that the integrand is harmonic as a function of $r$ and $t$ (see Baernstein [1, p. 153]). Clearly $I_{r}\left(\theta_{1}, \theta_{2}\right)>-\pi$ for all $0<\theta_{2}-\theta_{1}<2 \pi$ if and only if $G_{\phi}\left(r e^{i \tau}\right)>-\pi$ for all $\phi \in R, 0<r<1$, and $0<\tau<\pi$. The latter condition holds by the maximum principle for harmonic functions (Conway [4, p. 254]) when we show that for any fixed $\phi$, and all $a$ in the boundary of the upper half disc, $\liminf _{z \rightarrow a} G_{\phi}(z) \geqslant-\pi$.

We examine this limit in each of the four cases: $|a|=1 ; 0<a<1 ;-1<a<0$; $a=0$. For $a=e^{i \tau}$ we have

$$
\liminf _{z \rightarrow a} G_{\phi}(z)=\liminf _{\substack{r \rightarrow 1^{-} \\ \mu \rightarrow \tau}} G_{\phi}\left(r e^{i \mu}\right) \geqslant J(\phi-\tau, \phi+\tau) \geqslant-\pi,
$$

where the first inequality is by the definition of $J$ and the second by hypothesis. If $0<a<1$,

$$
\liminf _{z \rightarrow a} G_{\phi}(z)=\lim _{\substack{r \rightarrow a \\ \tau \rightarrow 0}} G_{\phi}\left(r e^{i \tau}\right)=\int_{\phi}^{\phi} F\left(f, \alpha, \beta ; r e^{i t}\right) d t=0>-\pi .
$$

If $-1<a<0$, using the Mean Value Theorem for harmonic functions (Conway [4, p. 253]) and the fact that $F(f, \alpha, \beta ; 0)=\alpha$, we have

$$
\liminf _{z \rightarrow a} G_{\phi}(z)=\lim _{\substack{r \rightarrow-a \\ \tau \rightarrow \pi}} G_{\phi}\left(r e^{i \tau}\right)=\int_{\phi-\pi}^{\phi+\pi} F\left(f, \alpha, \beta ; r e^{i t}\right) d t=2 \pi \alpha>0>-\pi .
$$


If $a=0$,

$$
\liminf _{z \rightarrow a} G_{\phi}\left(r e^{i \tau}\right)=\inf _{0 \leqslant \tau \leqslant \pi} 2 \tau \alpha=0>-\pi .
$$

This completes the proof of the lemma.

The main theorem. We now determine the range of $\alpha$ 's for which $f\left(z ; \psi_{1}, \psi_{2}\right)$ is $(\alpha, 0)$ Bazilevič.

THEOREM 2. $f\left(z ; \psi_{1}, \psi_{2}\right) \in B(\alpha, 0)$ if and only if

$$
\psi_{1} / \psi_{2} \leqslant \alpha-1 \leqslant\left(\pi / 2-\psi_{1}\right) /\left(\pi / 4-\psi_{2}\right) .
$$

Also, $f\left(z ; \psi_{1}, \psi_{2}\right)$ is Bazilevič ( for some $\left.(\alpha, \beta), \alpha>0\right)$ if and only if $\psi_{1} \leqslant 2 \psi_{2}$.

Proof. For keyhole $f\left(z ; \psi_{1}, \psi_{2}\right)$, let $J\left(\theta_{1}, \theta_{2}\right)$ be defined as in (5). We note that if neither $f\left(e^{i \theta_{1}}\right)$ nor $f\left(e^{i \theta_{2}}\right)$ is a vertex of the polygon, then the limit actually exists in (5) and

$$
J\left(\theta_{1}, \theta_{2}\right)=(\alpha-1)\left(\arg f\left(e^{i \theta_{2}}\right)-\arg f\left(e^{i \theta_{1}}\right)\right)+\sum e_{k},
$$

where $\arg f\left(r e^{i \theta}\right)$ is defined continuously for $\theta_{1} \leqslant \theta \leqslant \theta_{2}$, and the summation is over all $k$ for which the vertex $A_{k}$ is on the curve $f\left(e^{i \theta}\right), \theta_{1}<\theta<\theta_{2}$. By an analysis near points whose image is a vertex, we can verify that (7) also holds if $f\left(e^{i \theta_{1}}\right)$ or $f\left(e^{i \theta_{2}}\right)$ is a vertex, provided we take the sum over all $k$ with $A_{k}$ on $f\left(e^{i \theta}\right), \theta_{1} \leqslant \theta \leqslant \theta_{2}$, replacing $e_{k}$ by $\min \left\{0, e_{k}\right\}$ if $A_{k}=f\left(e^{i \theta_{1}}\right)$ or $A_{k}=f\left(e^{i \theta_{2}}\right)$.

We see that $\partial J / \partial \theta_{2}$ is positive when $f\left(e^{i \theta_{2}}\right)$ is on the open segment $\overline{A_{j}{ }_{1} A_{j}}$ and $j=1,3,5,6,7,8$ and negative when $f\left(e^{i \theta_{2}}\right)$ is on the segment for $j=2,4$. Also for fixed $\theta_{1}$, if $f\left(e^{i \omega}\right)=A_{k}$, the jump of $J\left(\theta_{1}, \theta_{2}\right)$ at $\theta_{2}=\omega$ is $e_{k}$. Since $J$ is lower semicontinuous, when we examine the signs of the $e_{k}$ 's as in (3), we see that for fixed $\theta_{1}, J\left(\theta_{1}, \theta_{2}\right)$ may have a local minimum only when $f\left(e^{i \theta_{2}}\right)=A_{2}$ or $A_{4}$. A similar analysis gives that for $\theta_{2}$ fixed, $J\left(\theta_{1}, \theta_{2}\right)$ may have a local minimum only when $f\left(e^{i \theta_{1}}\right)=A_{1}$ or $A_{3}$. Now suppose $\omega_{k}$ is such that $f\left(e^{i \omega_{k}}\right)=A_{k}$ for $0 \leqslant \omega_{k} \leqslant 2 \pi$ for $k=1, \ldots, 8$. Then we have $\min _{0 \leqslant \theta_{2}-\theta_{1} \leqslant 2 \pi} J\left(\theta_{1}, \theta_{2}\right)$ is the minimum of the four numbers $J\left(\omega_{1}, \omega_{2}\right), J\left(\omega_{1}, \omega_{4}\right), J\left(\omega_{3}, \omega_{4}\right)$ and $J\left(\omega_{3}, \omega_{2}+2 \pi\right)$. We now compute, using (7),

$$
\begin{gathered}
J\left(\omega_{1}, \omega_{2}\right)=e_{2}+(\alpha-1)\left(3 \pi / 4-\left(\pi-\psi_{2}\right)\right) \\
=-\pi / 2-\psi_{1}+(\alpha-1)\left(-\pi / 4+\psi_{2}\right), \\
J\left(\omega_{1}, \omega_{4}\right)=e_{2}+e_{3}+(\alpha-1)\left(\left(\pi+\psi_{2}\right)-\left(\pi-\psi_{2}\right)\right) \\
=-\pi-2 \psi_{1}+(\alpha-1)\left(2 \psi_{2}\right), \\
J\left(\omega_{3}, \omega_{2}+\pi\right)=\sum_{j=1}^{8} e_{j}+(\alpha-1)\left(\frac{11 \pi}{4}-\frac{5 \pi}{4}\right)=2 \pi+(\alpha-1)\left(\frac{3 \pi}{2}\right), \\
J\left(\omega_{3}, \omega_{4}\right)=J\left(\omega_{1}, \omega_{2}\right) .
\end{gathered}
$$

Now since $J\left(\omega_{3}, \omega_{2}+\pi\right)>-\pi$, the minimum of the four numbers above is greater than or equal to $-\pi$ if and only if $J\left(\omega_{1}, \omega_{2}\right) \geqslant-\pi$ and $J\left(\omega_{1}, \omega_{4}\right) \geqslant-\pi$. These two inequalities yield relationship (6) of the theorem, and thus the first part of the theorem follows from Theorem 1 and Lemma 2. 
To see the second part of the theorem, note that if $f\left(z ; \psi_{1}, \psi_{2}\right) \in B(\alpha, \beta)$ then, by Lemma 1 and symmetry, $f\left(z ; \psi_{1}, \psi_{2}\right) \in B(\alpha,-\beta)$. Since $\{(\alpha, \beta) \mid f(z) \in B(\alpha, \beta)\}$ is convex, this implies $f(z) \in B(\alpha, 0)$. Thus, by the first part of the theorem we must have $\left(\left(\pi / 2-\psi_{1}\right) /\left(\pi / 4-\psi_{2}\right)\right)-\psi_{1} / \psi_{2}$ nonnegative, which holds if and only if $2 \psi_{2} \geqslant \psi_{1}$.

Some remarks about the $\alpha$-projection. The second part of our main theorem establishes that if $\psi_{1} \leqslant 2 \psi_{2}$ then $f\left(z ; \psi_{1}, \psi_{2}\right)$ has nonempty $\alpha$-projection $[a, b]$, $a \leqslant b$, where

$$
a=\left(\psi_{1} / \psi_{2}\right)+1, \quad b=\left(\left(\pi / 2-\psi_{1}\right) /\left(\pi / 4-\psi_{2}\right)\right)+1 .
$$

For $\psi_{1} \leqslant 2 \psi_{2}$, using (8), we see $a \leqslant 3 \leqslant b$. Thus we have

COROLlaRY 1. $f\left(z ; \psi_{1}, \psi_{2}\right) \in B(\alpha, \beta)$ for some $(\alpha, \beta)$ only if $f\left(z ; \psi_{1}, \psi_{2}\right) \in B(3,0)$.

COROLlaRY 2. The $\alpha$-projection of a keyhole function is $\alpha=3$ if and only if $\psi_{1}=2 \psi_{2}$.

From (8) we also note that if $a=3$ then $b=3$, so the only intervals which may be $\alpha$-projections for keyhole functions satisfy $a \leqslant 3 \leqslant b$. Conversely, given an interval $[a, b], a \leqslant 3 \leqslant b$, we can exhibit the condition on $\psi_{1}, \psi_{2}$, hence, the specific keyhole function, for which a given interval is the $\alpha$-projection.

COROllaRY 3. The intervals $[a, b], a \neq b$, which are $\alpha$-projections of keyhole Bazilevič functions satisfy $1<a<3, b>3$. Every such interval is the $\alpha$-projection of the $f\left(z ; \psi_{1}, \psi_{2}\right)$ parameterized by

$$
\psi_{1}=\frac{\pi}{4}((a-1)(b-3)) /(b-a), \quad \psi_{2}=\psi_{1} /(a-1) .
$$

The equations (9) follow directly from (8).

Specification of allowable values for any two of $\psi_{1}, \psi_{2}, a, b$ as used above allows for computation of the other two using (8) and (9). For example, the interval form when $0<\psi_{1}=\psi_{2}<\pi / 4$ is $\left[2,3+\left(\psi_{1} /\left(\pi / 4-\psi_{1}\right)\right)\right]$, so for $\psi_{1}=\psi_{2}=\pi / 8$, the interval for which the function is $(\alpha, 0)$ Bazilevič is [2,4] and, in general, as $\psi_{1}=\psi_{2} \rightarrow \pi / 4$, then $b \rightarrow \infty$, and as $\psi_{1}=\psi_{2} \rightarrow 0$, then $b \rightarrow 3$.

\section{REFERENCES}

1. A. Baernstein II, Integral means, univalent functions and circular symmetrization, Acta Math. 133 (1974), 139-169.

2. I. E. Bazilevič, On a case of integrability in quadratures of the Löwner-Kufarev equation, Mat. Sb. 37 (1955), 471-476. (Russian)

3. D. Campbell and K. Pearce, Generalized Bazilevič functions, Rocky Mountain J. Math. 9 (1979), 197-226.

4. J. B. Conway, Functions of one complex variable, Springer-Verlag, New York, 1978

5. Z. Nehari, Introduction to complex analysis, rev. ed., Allyn and Bacon, Boston, Mass., 1968.

6. J. C. Plaster, A simple geometric criterion for non-Bazilevičness, Proc. Amer. Math. Soc. (to appear).

7. T. Sheil-Small, On Bazilevič functions, Quart., J. Math. Oxford Ser. (2) 23 (1972), 135-42.

Department of Mathematics and Computer Science, The Florida State University, TallahasSEE, FLORIDA 32306 